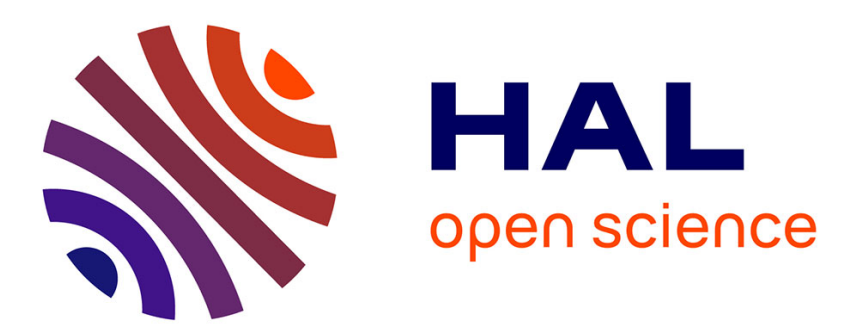

\title{
Thermal radiation of magma ocean planets using a 1-D radiative-convective model of $\mathrm{H} 2 \mathrm{O}-\mathrm{CO} 2$ atmospheres
} Emmanuel Marcq, Arnaud Salvador, Hélène Massol, Anne Davaille

\section{To cite this version:}

Emmanuel Marcq, Arnaud Salvador, Hélène Massol, Anne Davaille. Thermal radiation of magma ocean planets using a 1-D radiative-convective model of H2O-CO2 atmospheres. Journal of Geophysical Research. Planets, 2017, 122 (7), pp.1539-1553. 10.1002/2016JE005224 . insu-01564674

\section{HAL Id: insu-01564674 \\ https://hal-insu.archives-ouvertes.fr/insu-01564674}

Submitted on 13 Jan 2021

HAL is a multi-disciplinary open access archive for the deposit and dissemination of scientific research documents, whether they are published or not. The documents may come from teaching and research institutions in France or abroad, or from public or private research centers.
L'archive ouverte pluridisciplinaire HAL, est destinée au dépôt et à la diffusion de documents scientifiques de niveau recherche, publiés ou non, émanant des établissements d'enseignement et de recherche français ou étrangers, des laboratoires publics ou privés. 


\section{Journal of Geophysical Research: Planets}

\section{RESEARCH ARTICLE \\ 10.1002/2016JE005224 \\ Thermal radiation of magma ocean planets using a 1-D radiative-convective model of $\mathrm{H}_{2} \mathrm{O}-\mathrm{CO}_{2}$ atmospheres}

\section{Key Points:}

- Thermal blanketing confirmed at almost equal to $280 \mathrm{~W} / \mathrm{m}^{2}$, for steam-dominated atmospheres (Nakajima's limit) around magma ocean planets

- Thermal blanketing effective only for surface temperatures lower than a threshold, depending mainly upon the atmospheric water content - Detectability prospects for hot extrasolar magma ocean planets orbiting red dwarfs are optimal (contrast over 10\%) near 4 micrometer

Correspondence to:

E. Marcq,

emmanuel.marcq@latmos.ipsl.fr

Citation:

Marcq, E., A. Salvador, H. Massol, and A. Davaille (2017), Thermal radiation of magma ocean planets using a 1-D radiative-convective model of $\mathrm{H}_{2} \mathrm{O}-\mathrm{CO}_{2}$ atmospheres, $J$. Geophys. Res. Planets, 122, 1539-1553, doi:10.1002/2016JE005224.

Received 28 NOV 2016 Accepted 22 JUN 2017 Accepted article online 27 JUN 2017 Published online 26 JUL 2017

(O2017. American Geophysical Union. All Rights Reserved.

\author{
E. Marcq ${ }^{1}\left(\mathbb{D}\right.$, A. Salvador ${ }^{2,3}$, H. Massol ${ }^{3}$, and A. Davaille ${ }^{2}$ \\ ${ }^{1}$ LATMOS/IPSL, Université Paris-Saclay, Guyancourt, France, ${ }^{2}$ FAST, CNRS/Université Paris Sud, Université Paris-Saclay, \\ Orsay, France, ${ }^{3}$ GEOPS, Université Paris-Saclay, Orsay, France
}

\begin{abstract}
This paper presents an updated version of the simple 1-D radiative-convective $\mathrm{H}_{2} \mathrm{O}-\mathrm{CO}_{2}$ atmospheric model from Marcq (2012) and used by Lebrun et al. (2013) in their coupled interior-atmosphere model. This updated version includes a correction of a major miscalculation of the outgoing longwave radiation (OLR) and extends the validity of the model ( $P$ coordinate system, possible inclusion of $\mathrm{N}_{2}$, and improved numerical stability). It confirms the qualitative findings of Marcq (2012), namely, (1) the existence of a blanketing effect in any $\mathrm{H}_{2} \mathrm{O}$-dominated atmosphere: the outgoing longwave radiation (OLR) reaches an asymptotic value, also known as Nakajima's limit and first evidenced by Nakajima et al. (1992), around $280 \mathrm{~W} / \mathrm{m}^{2}$ neglecting clouds, significantly higher than our former estimate from Marcq (2012). (2) The blanketing effect breaks down for a given threshold temperature $T_{\varepsilon}$, with a fast increase of OLR with increasing surface temperature beyond this threshold, making extrasolar planets in such an early stage of their evolution easily detectable near $4 \mu \mathrm{m}$ provided they orbit a red dwarf. $T_{\varepsilon}$ increases strongly with $\mathrm{H}_{2} \mathrm{O}$ surface pressure, but increasing $\mathrm{CO}_{2}$ pressure leads to a slight decrease of $T_{\varepsilon}$. (3) Clouds act both by lowering Nakajima's limit by up to $40 \%$ and by extending the blanketing effect, raising the threshold temperature $T_{\varepsilon}$ by about $10 \%$.
\end{abstract}

Plain Language Summary Recently formed Earth-sized planets experience a "magma ocean" stage, where molten rocks extend from the core up to the surface. These planets are able to cool themselves by radiating more heat through their thick atmospheres than they absorb from their parent star. We have investigated the effect of the total atmospheric content (assumed to consist mostly of water vapor and carbon dioxide) and of the surface temperature of the magma ocean upon the rapidity of the cooling. Our main finding is that there are two stages: for very high surface temperatures, cooling is fast, and only thin clouds can form. Such planets would be quite easily detected since they radiate very efficiently in the infrared range. Conversely, relatively cool surface temperatures lead to cooler upper atmospheres, harboring thick water clouds. Such planets would be very difficult to distinguish from more mature planets such as Earth or Venus from the point of view of a remote observer.

\section{Introduction}

Young telluric planets are expected to harbor very different atmospheres compared to those we already know about. Even restricting the field of study to only secondary atmospheres - so after the lightest elements ( $\mathrm{He}$ and $\mathrm{H}_{2}$ ) have escaped - the $10^{7}$ years or more required to significantly alter the composition of $\mathrm{C}_{-}, \mathrm{N}-$, or $\mathrm{O}-$ bearing volatiles through atmospheric escape may result in much heavier atmospheres than even present-day Venus. Another specificity of such young planets is that their internal heat flux is several orders of magnitude larger than more mature planets like present-day Earth, for example, and can be comparable to the stellar fluxes while in their magma ocean stage; this pushes these atmospheres out of global radiative balance, making them radiate significantly more energy to outer space than they absorb from their host stars. Since most atmospheric models assume a negligible geothermal heat flux, they cannot be used here. Furthermore, since the interaction between these early atmospheres with both the interior and escape processes is significant, specific coupled models are required to proceed.

However, very dense telluric atmospheres are also expected in other situations, namely, when modeling runaway atmospheres: these atmospheres usually include a full Earth ocean equivalent ( 300 bar) as atmospheric water vapor. It is therefore of no surprise that some of the earlier models partially relevant for our studies deal 
with such atmospheres [Komabayashi, 1967; Ingersoll, 1969; Kasting, 1988; Nakajima et al., 1992]. In the context of exoplanetary studies, investigating the inner, runaway-limited edge of the habitability zone has received a lot of recent attention using general circulation models or simple 1-D columns [Leconte et al., 2013; Goldblatt et al., 2013; Kopparapu et al., 2013; Yang et al., 2014; Yang and Abbot, 2014; Hamano et al., 2015; Kopparapu et al., 2016].

Recently outgassed atmospheres around magma ocean planets and out of global radiative balance were first numerically investigated in the 1980s [Abe and Matsui, 1988]. Such models are still being developed nowadays [Elkins-Tanton, 2008; Marcq, 2012; Lebrun et al., 2013; Hamano et al., 2013, 2015; Lupu et al., 2014; Schaefer et al., 2016], with various levels of complexity or coupling. We aim to present here the most recent updates to the 1-D radiative-convective model of Marca [2012] used by Lebrun et al. [2013] and Salvador et al. [2017]. We will first detail our improvements in section 2 . In section 3, we will review our updated results, and how they bear on the detectability of magma ocean planets. Finally, we will conclude and discuss the limitations of our model in section 4.

\section{Model Description}

\subsection{Thermal Structure}

As in Marca [2012], up to three different physical layers (not to be confused with the computational layers, which are typically a few hundred) are possible in this model. From the bottom up (as can be seen in Figures 7 and 9), they are, namely, the following: (1) unsaturated troposphere, (2) moist troposphere, and (3) mesosphere. Moist troposphere is present only if the saturation of water vapor is reached at any altitude-if this occurs at the surface, then unsaturated troposphere will not exist, and any water in excess of $100 \%$ saturation is assumed to condense and form a water ocean overlying the magma ocean.

\subsubsection{Unsaturated Troposphere}

This lowermost convective layer follows, as in Marcq [2012], a dry adiabatic lapse rate. In terms of the new pressure coordinate, $(\partial T / \partial P)_{S}$ follows Kasting [1988]:

$$
(\partial T / \partial P)_{S}=\left[\rho_{v} T\left(\partial V_{v} / \partial T\right)_{P}\right] /\left[\rho_{v} C_{P, v}(T)+\rho_{c} C_{P, c}(T)+\rho_{o} C_{P, o}(T)\right]
$$

where $V_{v}=1 / \rho_{v}$ is the specific volume of water vapor.

The thermodynamical properties of water vapor are computed using the Fortan NBS/NRC steam tables [Haar et al., 1984], which implies that $\mathrm{H}_{2} \mathrm{O}$ is not considered as an ideal gas in our model - this is required since the critical point of water lies within the range of modeled pressures and temperatures. $\mathrm{CO}_{2}$ and $\mathrm{N}_{2}$, on the other hand, are always considered as ideal gases since the $(T, P)$ range of our model lies very far from their critical points. $C_{P, c}(T)$ for $\mathrm{CO}_{2}$ follows the expression from Abe and Matsui [1988]. $C_{P, o}(T)$ is approximated by the analytic expression $C_{P, o}(T)=1040+488\left(e^{-688.1 \mathrm{~K} / T}\right)^{2}$ in $\mathrm{J} / \mathrm{kg} / \mathrm{K}$, fitted in the 300 to $2500 \mathrm{~K}$ temperature range from Chase [1998] National Institute of Standards and Technology data.

\subsubsection{Moist Troposphere}

This layer is still convective, but $\mathrm{H}_{2} \mathrm{O}$ condensation takes place so that the lapse rate is now given by the following:

$$
(\partial T / \partial P)_{S}=\left[\left(d P_{\text {sat }} / d T\right)+\rho_{n} R / M_{n}\left(1+\partial \ln \rho_{v} / \partial \ln T-\partial \ln \alpha_{v} / \partial \ln T\right)\right]^{-1}
$$

Here $\rho_{n}=\rho_{c}+\rho_{o}$, and $M_{n}$ stands for the weighted molecular mass of the non condensible $\mathrm{CO}_{2}-\mathrm{N}_{2}$ mixture. Following Kasting [1988], we define $\alpha_{v}=\rho_{v} / \rho_{n}$, and its derivative $\partial \ln \alpha_{v} / \partial \ln T$ is computed according to the formula given in Marcq [2012] and Kasting [1988]:

$$
\partial \ln \alpha_{v} / \partial \ln T=\frac{R / M_{n}\left(\partial \ln \rho_{v} / \partial \ln T\right)-C_{v, n}(T)-\alpha_{v}\left(\partial s_{v} / \partial \ln T\right)}{\alpha_{v}\left(s_{v}-s_{c}\right)+R / M_{n}}
$$

with $s_{c}$ standing for the specific entropy of condensed water.

Wordsworth and Pierrehumbert [2013] recently showed that the above expression is only valid when both water vapor and the $\mathrm{CO}_{2}-\mathrm{N}_{2}$ mixture can be considered as ideal gases. Although, strictly speaking, this is not the case in our model regarding $\mathrm{H}_{2} \mathrm{O}$, water condensation usually occurs at higher altitudes, and therefore 
relatively low pressure and temperature, far from the critical point of water. Departure between our $\mathrm{H}_{2} \mathrm{O}$ thermodynamical tables and ideal gas was empirically found to be less than a few percents in these layers, so that we could still make use of the derivation from Kasting [1988] in most situations.

\subsubsection{Mesosphere}

Since our model does not include yet any radiative transfer for the incoming short wave stellar radiation, we are unable to reproduce any stratosphere (defined as a radiative, nonconvective layer where temperature increases with increasing height). Therefore, we call mesosphere the radiative, nonconvective layer on top of the troposphere. The base of our mesosphere (the tropopause) is reached once the tropospheric temperature falls below a given temperature $T_{0}$. Although the local radiative equilibrium would allow an iterative calculation of the mesospheric temperature profile, its integrated Rosseland opacity was empirically found to be thin. Our simple model therefore considers this mesosphere as an isothermal layer at the temperature $T_{0}$, and thus $d T / d P=0$ there. $\alpha_{v}$ is also considered vertically uniform in this layer. However, it is noteworthy that real gas effects may lead to strong departures from this prescribed isothermal profile, but a more realistic computation of the radiative temperature profile would require the inclusion of stellar radiation.

$T_{0}$ can therefore be understood as the top of atmosphere (TOA) temperature of our model. Kasting [1988] sets it to $200 \mathrm{~K}$, but our model is intended to apply to even hotter atmospheres. There are two options for the final user here: (1) fix $T_{0}$ to a constant temperature; standard choices are $200 \mathrm{~K}$ for an $\mathrm{H}_{2} \mathrm{O}$-rich mesosphere as Kasting [1988] did, or even colder as long as $\mathrm{CO}_{2}$ is a major constituent. For example, TOA temperature is close to $150 \mathrm{~K}$ for [Leconte et al., 2013], and this cold TOA temperature issue is also extensively discussed by Wordsworth and Pierrehumbert [2013] — let us also mention that at the very top of our model, NLTE (nonlocal thermodynamical equilibrium) effects could kick in for $\mathrm{CO}_{2}$ radiative cooling so that even the most precise line-by-line LTE calculations would not be accurate. The second option (2) is to adjust iteratively $T_{0}$ so that the relative divergence of outgoing longwave radiation (OLR) at the top of our model $\left(P=0.1 \mathrm{~Pa}, \tau_{i} \sim 0\right)$ :

$$
\left|\frac{\sum_{i \in \text { bands }} k_{\mathrm{abs}, \mathrm{i}} \partial \mathrm{OLR}_{\mathrm{i}} / \partial \tau_{i}}{\sum_{i \in \text { bands }} k_{\mathrm{abs}, \mathrm{i}} \mathrm{OLR}_{i}}\right|<0.02
$$

This adjustment results in very cold mesosphere ( $T_{0}$ around $150 \mathrm{~K}$ or less) even when the surface temperature is very hot. Since Kasting [1988] found that total OLR was not much dependent on his prescribed value $T_{0}$ as long as $T_{0}<240 \mathrm{~K}-\mathrm{cool}$ mesospheres do not contribute much to the thermal radiation. Therefore, since we do not include yet any radiative stellar heating of these layers (that could dramatically rise their temperature) and following similar temperature profiles from Lupu et al. [2014], we hereafter adopt a fixed value $T_{0}=200 \mathrm{~K}$.

\subsection{Radiative Calculations}

\subsubsection{Short Wave Radiation}

Shortwave radiation is not taken into account in this model, as in Marcq [2012]. Its effect on the radiative budget of the planet is parameterized through a bolometric albedo $A$, so that the absorbed stellar radiation is computed as $(1-A) F_{*}$ where $F_{*}$ is the integrated stellar constant. In this version of the model, $A$ is computed as $A=A_{\text {clear }}\left(1-e^{-\tau_{c}}\right)+A_{\text {cloud }} e^{-\tau_{c}}$ where $\tau_{c}$ stands for the optical depth of the clouds (when present and considered) in the visible range - this enables a smooth transition when clouds appear and thus avoids any threshold effect. Since the temperature profile is not computed from the radiative fluxes (except optionally for $T_{0}$, see section 2.1.3), this crude parameterization is valid to estimate the radiative balance of the planet. Please note that this albedo parameterization is only used in coupled interior-atmosphere simulations and is irrelevant when discussing the sole atmospheric subsystem, as is the case in this paper.

The former version of the model used different values for $A_{\text {clear }}=0.2$ (Mars like) and $A_{\text {clouds }}=0.7$ (Venus like); however, recent progress in dense atmosphere radiative transfer modeling [Leconte et al., 2013; Hamano et al., 2015; Kopparapu et al., 2013; Goldblatt et al., 2013] showed that the albedo of $\mathrm{H}_{2} \mathrm{O}$-rich atmospheres is always quite low, even when thick clouds are present. Therefore, following Leconte et al. [2013] who unlike other studies took into account the radiative effect of clouds, the coupled simulations of Salvador et al. [2017] primarily use $A_{\text {clear }}=A_{\text {cloud }}=0.2$, although the final user can still choose different values for $A_{\text {clear }}$ and $A_{\text {cloud }}$. The albedo could however be substantially higher (equal or above 0.5) for $\mathrm{CO}_{2}$-rich, $\mathrm{H}_{2} \mathrm{O}$-poor atmospheres [Kopparapu et al., 2013, their Figure 5] or for slow rotating planets exhibiting permanent convective clouds near the substellar point [Yang et al., 2013]. This further highlights the need for an improved short wave radiation scheme in a future version of our model (see section 4.1.2). 


\subsubsection{Long Wave Radiation}

In our model, the thermal radiation encompasses wave numbers between 0 and $10,100 \mathrm{~cm}^{-1}$. This thermal radiation can be computed either (1) following the gray approximation in the aforementioned range or (2) using a $k$ correlated radiative transfer code. The former yields cruder estimates of the resulting OLR, but is much faster, and can therefore be used in first, exploratory simulations where no spectroscopic output is needed. We use the discrete ordinates radiative transfer (DISORT) [Stamnes et al., 1988] solver with four streams in the whole atmosphere, so that scattering (e.g., by clouds, see after) can be taken into account. We now discuss the sources of long wave opacity provided as inputs to DISORT.

In the gray approximation, the mass absorption coefficients of $\mathrm{H}_{2} \mathrm{O}$ and $\mathrm{CO}_{2}$ are, respectively, set equal to $10^{-2}$ and $10^{-4} \mathrm{~m}^{2} / \mathrm{kg}$ following Nakajima et al. [1992]. Our $\mathrm{k}$-correlated code uses the same high-resolution spectrum (truncated at $100 \mathrm{~cm}^{-1}$ to avoid any overlap with continuum opacity, see below) computed by KSPECTRUM [Eymet et al., 2016] as Leconte et al. [2013] and also shares the same choice of $16 \mathrm{~g}$ values. The set of corresponding $16 k$ coefficients is then stored in a three-dimensional look-up table $\left(\mathrm{H}_{2} \mathrm{O} / \mathrm{CO}_{2}\right.$ ratio, temperature, and total pressure including $\mathrm{N}_{2}$ ) suitable for fast interpolation. This extensive rewriting of the $k$-correlated code led us to correct a major mistake in the first published version of Marcq [2012] that resulted in a severe underestimation of the resulting OLR by a factor of about 2 . Therefore, the atmosphere and the coupled magma ocean should be cooling faster than previously reported by Lebrun et al. [2013]. This will be further discussed in section 3.4.

Continuum opacity is calculated and added in both gray and $k$-correlated cases and is unchanged since Marca [2012]: $\mathrm{H}_{2} \mathrm{O}-\mathrm{H}_{2} \mathrm{O}$ continuum opacity is taken from Clough et al. [2005], and $\mathrm{CO}_{2}-\mathrm{CO}_{2}$ fitted from Venus observations by Bruno Bézard [Bézard et al., 2011; Marcq et al., 2008]. $\mathrm{H}_{2} \mathrm{O}-\mathrm{CO}_{2}$ continuum opacity is not considered, and in any case negligible compared to $\mathrm{H}_{2} \mathrm{O}-\mathrm{H}_{2} \mathrm{O}$ [Ma and Tipping, 1992, their Figure 7] and/or $\mathrm{CO}_{2}-\mathrm{CO}_{2}$ depending on the dominant species in the atmosphere.

\subsubsection{Clouds}

Clouds are considered present throughout the atmosphere wherever $\mathrm{H}_{2} \mathrm{O}$ saturation occurs, i.e., in the moist tropospheric layer. Their radiative effect is taken into account through albedo parameterization for the stellar radiation (see section 2.2.1), and in the radiative transfer code for the thermal radiation. Their optical properties and microphysical properties - mass loading, $\varpi_{0}(\lambda), g(\lambda)$, Henyey-Greenstein phase function, and $Q_{\text {ext }}(\lambda)$ - still follow the simple parameterization from Kasting [1988] and already used in Marcq [2012]. However, since a realistic modeling of the radiative budget of clouds is not possible in a 1-D atmospheric column model such as ours, the user can choose to disable the scattering of thermal radiation by these clouds. Also, we do not couple our atmospheric model to a microphysical model, so that our cloud layer parameterization probably overestimates their actual thickness - as an example, when running with present-day Earth setup, our model finds thick clouds extending in the whole troposphere, from the surface up to $10 \mathrm{~km}$, with an optical thickness close to 300 at $1 \mu \mathrm{m}$. So one can compute OLR with and without taking into account the radiative effects of the cloud cover, knowing that the actual OLR is bracketed between these two extreme situations. Also, it is worth noting that scattering and/or absorbing mesospheric photochemical aerosols (not included in our model) could lead to a further decrease in OLR as well as alter the temperature profile.

\section{Results}

\subsection{OLR Versus $T_{\text {surf }}$}

In most situations, water vapor is present in sufficient quantity (i.e., $P_{\text {surf }}\left(\mathrm{H}_{2} \mathrm{O}\right)>\left[P_{\text {surf }}\left(\mathrm{CO}_{2}\right) / 15 \text { bar }\right]^{1.6} \times 1$ bar according to Figure 5; see section 3.1.2 for further discussion) in the atmosphere. In such a case, the variation of spectrally integrated OLR with respect to the surface temperature displays the features visible in Figure 1. These common features are (\#1) the existence of an asymptotic regime at relatively low temperatures, where the OLR is quite low (the so-called blanketing effect) and exhibits very little dependency with surface temperature; (\#2) at higher surface temperatures, the OLR increases rapidly with surface temperature; (\#3) optically thick clouds have two main effects: (\#3a) they substantially lower the asymptotic OLR, and (\#3b) they extend the blanketing effect to higher surface temperatures - at very high surface temperatures, clouds become optically thinner and thinner; thus, both scenarios yield the same OLR.

Features \#1 and \#2 have been consistently reproduced by all similar radiative-convective models used for both models of atmospheres around magma ocean planets [Hamano et al., 2013; Marcq, 2012] or, more often, studying the runaway greenhouse effect [Kasting, 1988; Nakajima et al., 1992; Goldblatt et al., 2013]. In fact, 


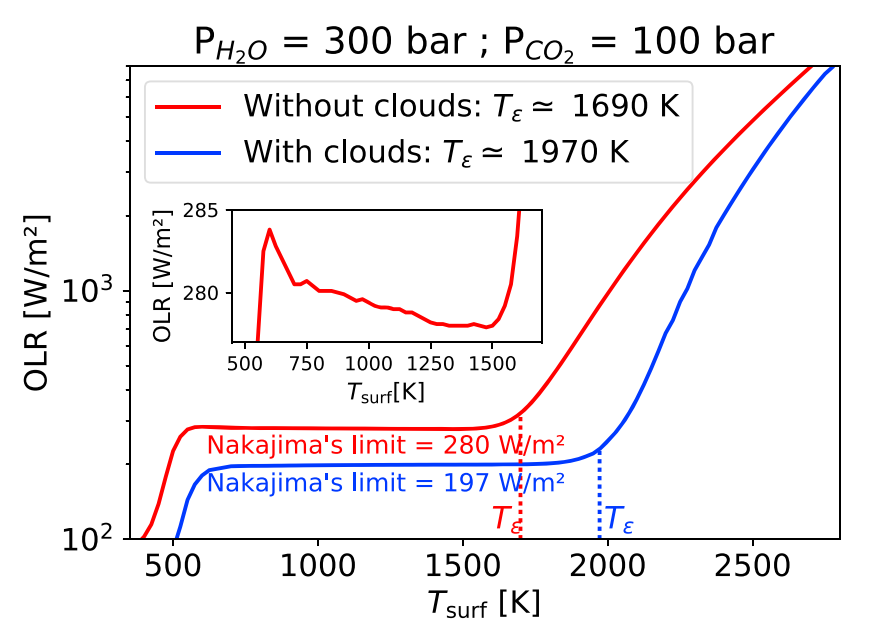

Figure 1. Outgoing longwave radiation $(\mathrm{OLR})$ versus surface temperature for a total volatile inventory $P_{\text {surf }}\left(\mathrm{H}_{2} \mathrm{O}\right)=$ 300 bar and $P_{\text {surf }}\left(\mathrm{CO}_{2}\right)=100$ bar neglecting (red) or taking into account (blue) the radiative effect of water clouds extending throughout the moist troposphere. The small decrease in OLR for $T_{\text {surf }}<T_{\varepsilon}$ is shown in insert.

the very existence of this near-asymptotic behavior of OLR versus $T_{\text {surf }}$ is responsible for the instability characterizing the runaway greenhouse effect: a tiny increase in absorbed solar radiation (and also of equal OLR when in global radiative balance) can trigger a rise of surface temperature by several hundreds of kelvins until global radiative balance is restored at a very high surface temperature. Actually, OLR decreases by a few $\mathrm{W} / \mathrm{m}^{2}$ with increasing surface temperatures below $1400 \mathrm{~K}$ for such atmospheres, as shown in the insert from Figure 1. This counterintuitive effect arises in $\mathrm{H}_{2} \mathrm{O}-\mathrm{CO}_{2}$ radiative-convective models as explained by Nakajima et al. [1992]: thermal brightness for a given wavelength is directly related to vertically integrated lapse rate $d T / d \tau$ above $\tau=1$, which usually lies within the moist troposphere. A hotter surface temperature will therefore move the moist troposphere upward to lower pressure levels, thus lowering the moist lapse rate. This OLR decrease results in an even more unstable situation as a straight plateau and can be a source of hysteresis in the context of interior-atmospheres models where coupling is performed using the internal heat flux [e.g., Salvador et al., 2017]: two very different surface temperatures yield the same OLR and therefore require the same internal heat flux.

In the context of magma ocean planets, such an efficient blanketing effect can be reached only for planets far enough from their host star (so-called Hamano-type I planets) and thus able to reach global radiative balance below this asymptotic value. This value is also known as Nakajima's limit (NL) since it was accurately described for the first time by Nakajima et al. [1992]. Further study of NL in our model and comparison with other works is found in section 3.1.2.

We can define formally the aforementioned blanketing effect through an effective emissivity $\varepsilon$ defined as OLR $=\sigma T_{\text {eff }}^{4}=\varepsilon \sigma T_{\text {surf }}^{4}$ - the smaller the value of $\varepsilon$, the stronger the blanketing effect. It appears (see Figure 2) that $\varepsilon$ exhibits a minimum with respect to $T_{\text {surf }}$ for a given value $T_{\text {surf }}=T_{\varepsilon}$. Thus, $T_{\varepsilon}$ provides a self-consistent and physically sound threshold to distinguish the low-temperature regime $T_{\text {surf }}<T_{\varepsilon}$ from the high-temperature regime $T_{\text {surf }}>T_{\varepsilon}$, even for $\mathrm{CO}_{2}$-rich atmospheres which do not exhibit the asymptotic regime at OLR $=\mathrm{NL}$ for $T_{\text {surf }}<T_{\varepsilon}$ (see Figure 3). The variations of $T_{\varepsilon}$ with respect to $\mathrm{H}_{2} \mathrm{O}$ and $\mathrm{CO}_{2}$ surface pressures will be addressed in section 3.1.1.

Features \#3a and \#3b are not reproduced by all models, and heavily dependent on the assumptions regarding the physical properties of the cloud layers. Kasting [1988] already performed a sensitivity study on the radiative effect of cloud layers depending on their vertical location and found their influence from moderate (decreasing OLR by a few tens of percents) to negligible. As previously discussed, our parameterization (clouds extending throughout the whole moist troposphere) yields the optically thickest scenario. Others have faced these problems before [Hamano et al., 2015; Kopparapu et al., 2013], and it appears that no satisfactory representation of clouds can be performed using a 1-D column model like ours - this is mainly due to the nonlinear radiative effects of clouds upon the OLR. Also, due the complexity of the involved microphysics, even 3-D models are unlikely to yield a satisfactory representation of the clouds in the near future. 


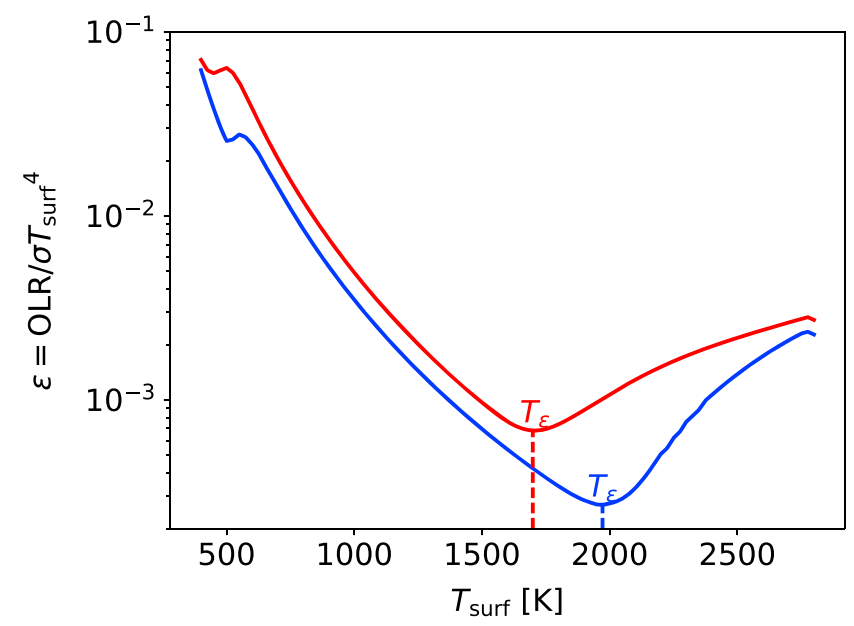

Figure 2. Graphical determination of $T_{\varepsilon}$ from $\varepsilon=f\left(T_{\text {surf }}\right)$ for both OLR plotted in Figure 1 .

\subsection{1. $T_{\varepsilon}$ Versus Atmospheric Inventory}

Figure 4 shows the behavior of threshold temperature $T_{\varepsilon}$ as defined in the previous section. As expected, the efficiency of the blanketing effect at higher surface temperatures is dominated by the total water vapor atmospheric content. This reflects the well-known fact that $\mathrm{H}_{2} \mathrm{O}$ is a much more efficient greenhouse gas than $\mathrm{CO}_{2}$.

Aside from the low $\mathrm{H}_{2} \mathrm{O}$-high $\mathrm{CO}_{2}$ situation — which does not actually exhibit asymptotic limit for $T_{\text {surf }}<T_{\varepsilon^{\prime}}$ see section 3.1.2- we can see that with a fixed atmospheric water vapor content, adding more $\mathrm{CO}_{2}$ in the atmosphere is actually detrimental to the blanketing effect. This may come as a surprise but can be understood as follows. As previously stated here and following Nakajima et al. [1992], the thermal brightness for a given wavelength is related to the vertically integrated lapse rate $\mathrm{d} T / \mathrm{d} \tau$ above the $\tau \sim 1$ level, which for most wavelengths is located in the higher troposphere. Adding more $\mathrm{CO}_{2}$ while keeping $\mathrm{H}_{2} \mathrm{O}$ the same results in a lower humidity ratio $\alpha_{v}$ in the troposphere, which in turn leads to a steeper adiabatic lapse rate (in terms of $\mathrm{d} T / \mathrm{d} \tau$, since $\mathrm{CO}_{2}$ mass absorption coefficient is much lower than for $\mathrm{H}_{2} \mathrm{O}$ ). This results in an increase of OLR for a given surface temperature, thus weakening the blanketing effect.

The variations of $T_{\varepsilon}$ for a large domain in 10 bar $<P_{\text {surf }}\left(\mathrm{H}_{2} \mathrm{O}\right)<300$ bar and $5 \mathrm{bar}<P_{\text {surf }}\left(\mathrm{CO}_{2}\right)<100$ bar according to Figure 4 are approximated by $\Delta T_{\varepsilon} / T_{\varepsilon} \approx 0.22 \Delta P_{\text {surf }}\left(\mathrm{H}_{2} \mathrm{O}\right) / P_{\text {surf }}\left(\mathrm{H}_{2} \mathrm{O}\right)-0.05 \Delta P_{\text {surf }}\left(\mathrm{CO}_{2}\right) / P_{\text {surf }}\left(\mathrm{CO}_{2}\right)$, which yields after integration an approximate analytical parameterization:

$$
T_{\varepsilon} \approx 1420 \mathrm{~K}\left[\frac{P_{\text {surf }}\left(\mathrm{H}_{2} \mathrm{O}\right)}{100 \mathrm{bar}}\right]^{0.22}\left[\frac{P_{\text {surf }}\left(\mathrm{CO}_{2}\right)}{30 \mathrm{bar}}\right]^{-0.05}
$$

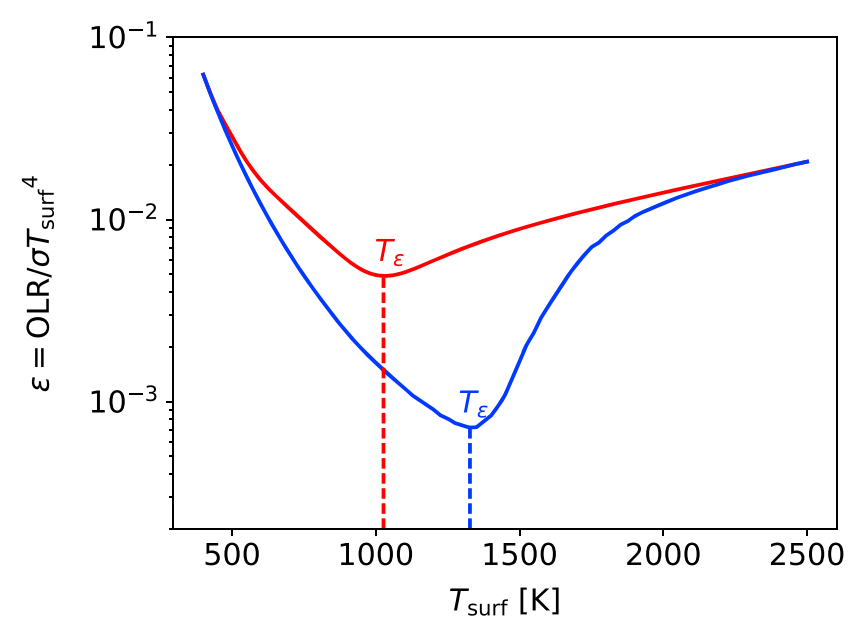

Figure 3. Graphical determination of $T_{\varepsilon}$ from $\varepsilon=f\left(T_{\text {surf }}\right)$ for both OLR plotted in Figure 6 . 


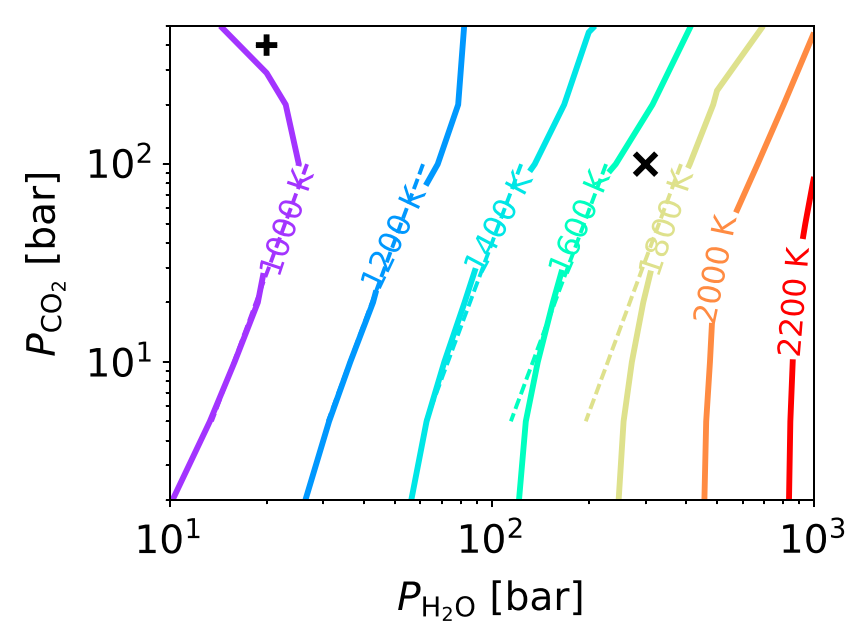

Figure 4. Contour plot of threshold temperature $T_{\varepsilon}$ versus surface pressures $P_{\text {surf }}\left(\mathrm{H}_{2} \mathrm{O}\right)$ and $P_{\text {surf }}\left(\mathrm{CO}_{2}\right)$. Cross sign indicates the atmospheric inventory from Figure 1, and plus sign the atmospheric inventory from Figure 6 . Dashed lines indicate the approximate analytical parameterization stated in section 3.1.1.

\subsubsection{Nakajima's Limit Versus Atmospheric Inventory}

Figure 5 shows the asymptotic Nakajima's limit, estimated here using OLR ( $T_{\text {surf }}=800 \mathrm{~K}$ ) since $800 \mathrm{~K}<T_{\varepsilon}$ in the investigated $\left(P_{\mathrm{H}_{2} \mathrm{O}}, P_{\mathrm{CO}_{2}}\right)$ parameter space. Its most striking feature is a sharp boundary (shown as a dashed line in Figure 5 between the $\mathrm{CO}_{2}$-dominated atmospheres in the upper left corner $\left(P_{\text {surf }}\left(\mathrm{H}_{2} \mathrm{O}\right)>\right.$ $\left[P_{\text {surf }}\left(\mathrm{CO}_{2}\right) / 15 \mathrm{bar}\right]^{1.6} \times 1$ bar, and $\mathrm{H}_{2} \mathrm{O}$-dominated atmospheres (everywhere else).

For $\mathrm{H}_{2} \mathrm{O}$-dominated atmospheres, $\mathrm{NL}$ is remarkably independent of the exact atmospheric composition and/or surface pressure, with values ranging between 275 and $283 \mathrm{~W} / \mathrm{m}^{2}$, well above Marcq [2012] uncorrected estimate of $220 \mathrm{~W} / \mathrm{m}^{2}$. This corrected value is in a very good agreement (within $2.5 \%$ ) with recent runaway greenhouse studies, whether using a line-by-line [Goldblatt et al., 2013] $\left(282 \mathrm{~W} / \mathrm{m}^{2}\right)$ or $k$-correlated [Leconte et al., 2013] (also $282 \mathrm{~W} / \mathrm{m}^{2}$ ) radiative transfer code. This qualitative behavior was also reproduced by older random band or gray models [Nakajima et al., 1992; Kasting, 1988], albeit with larger values for NL on the order of $300 \mathrm{~W} / \mathrm{m}^{2}$ or more. A detailed physical interpretation about how all steam-dominated atmospheres exhibit this same NL value can be found in Goldblatt et al. [2013]: as long as the mesosphere is cool enough, the $T(p)$ profile in the atmospheric layers that contribute to the OLR is mostly unchanged, although the altitude of these layers may vary greatly. The small variations of $\pm 2 \mathrm{~W} / \mathrm{m}^{2}$ we find for NL originate probably from the fact that we estimate the asymptotic NL with a more computationally robust proxy OLR $\left(T_{\text {surf }}=800 \mathrm{~K}\right)$ :

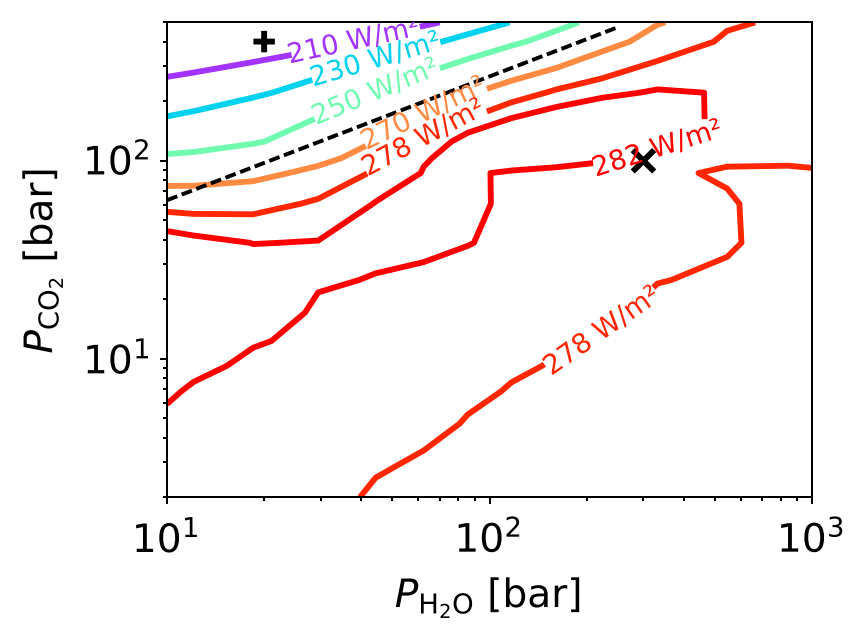

Figure 5. Contour plot of OLR ( $T_{\text {surf }}=800 \mathrm{~K}$ ) (indicative of Nakajima's limit value) versus surface pressures $P_{\text {surf }}\left(\mathrm{H}_{2} \mathrm{O}\right)$ and $P_{\text {surf }}\left(\mathrm{CO}_{2}\right)$. Cross sign indicates the atmospheric inventory from Figure 1, and plus sign the atmospheric inventory from Figure 6. A dashed line separates $\mathrm{H}_{2} \mathrm{O}$-dominated atmospheres from $\mathrm{CO}_{2}$-dominated atmospheres. 


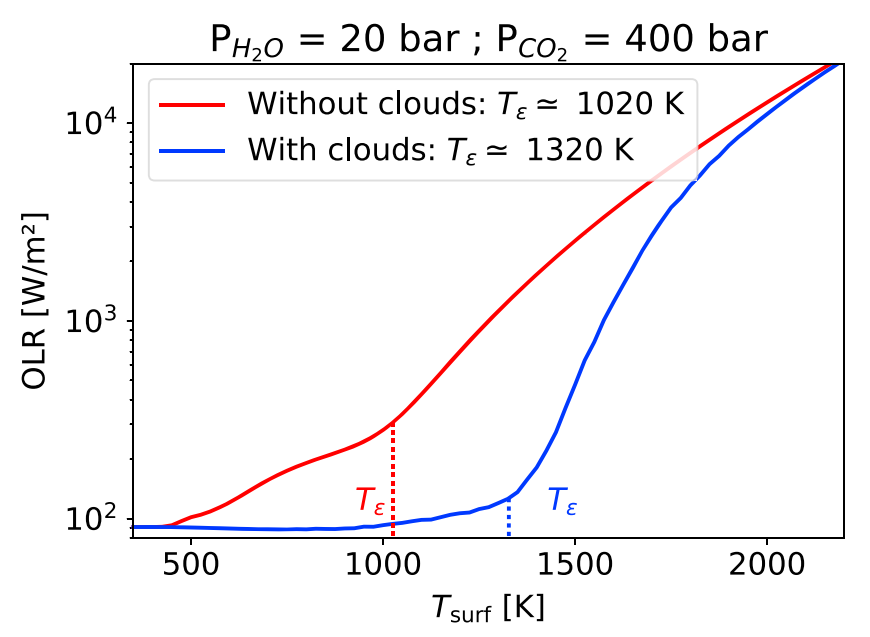

Figure 6. Outgoing longwave radiation (OLR) versus surface temperature for a total volatile inventory $P_{\text {surf }}\left(\mathrm{H}_{2} \mathrm{O}\right)=$ 20 bar and $P_{\text {surf }}\left(\mathrm{CO}_{2}\right)=400$ bar neglecting (red) or taking into account (blue) the radiative effect of water clouds extending throughout the moist troposphere.

there is indeed a local maximum for OLR $=f\left(T_{\text {surf }}\right)$ at relatively cool $T_{\text {surf }}$ values for $\mathrm{H}_{2} \mathrm{O}-\mathrm{CO}_{2}$ atmospheres as seen and discussed by Nakajima et al. [1992, their Figure 6], and the OLR at $T_{\text {surf }}=800 \mathrm{~K}$ might be influenced by this maximum and shifted then to slightly higher values compared to the actual NL.

On the other hand, for $\mathrm{CO}_{2}$-dominated atmospheres, the $\mathrm{OLR}$ at $T_{\text {surf }}=800 \mathrm{~K}$ is varying with the atmospheric composition and decreases strongly with increasing $\mathrm{CO}_{2}$ content (see Figure 5). Further investigations show that for these atmospheres, the function OLR $=f\left(T_{\text {surf }}\right)$ does not exhibit any asymptotic behavior as can be seen in Figure 1 but rather increases monotonically with increasing $T_{\text {surf }}$ as can be seen in Figure 6 - although effective emissivity $\varepsilon$ still displays a local minimum with respect to $T_{\text {surf }}$, so that our definition of $T_{\varepsilon}$ remains valid. Since $\mathrm{CO}_{2}$ partial pressure, unlike $\mathrm{H}_{2} \mathrm{O}$, is not governed by its saturated vapor pressure in radiatively active layers, the reasoning from Goldblatt et al. [2013] does not apply to these atmospheres, which accordingly cannot be said to be experiencing a runaway $\mathrm{H}_{2} \mathrm{O}$ greenhouse. It is therefore misleading to define a Nakajima's limit for these atmospheres. One could remark that the present-day Venusian atmosphere, with an OLR near $170 \mathrm{~W} / \mathrm{m}^{2}$ for $P_{\text {surf }}\left(\mathrm{CO}_{2}\right)=92$ bar and $P_{\text {surf }}\left(\mathrm{H}_{2} \mathrm{O}\right) \approx 3 \mathrm{mbar}$, is fully representative of this $\mathrm{CO}_{2}$-dominated regime although we did not perform simulations with such a low water abundance. Let us finally note that a $\mathrm{CO}_{2}$-dominated atmosphere is not expected for a recently outgassed atmosphere prior to differential atmospheric escape, so that we will restrict the discussion in the next sections to more typical $\mathrm{H}_{2} \mathrm{O}$-dominated atmospheres.

\subsection{Vertical Structure and Low Resolution OLR Spectra}

3.2.1. $T_{\text {surf }}<T_{\varepsilon}$

A typical atmospheric structure for $\mathrm{H}_{2} \mathrm{O}$-dominated atmospheres in the $T_{\text {surf }}<T_{\varepsilon}$ regime is shown in Figure 7 . The three-stage structure is well visible here, and in some way reminiscent of an exaggerated present-day Venus: from the bottom up, a clear unsaturated layer with no possible condensation, then a saturated layer where condensation may occur over several scale heights and form thick clouds, underneath a relatively cool $(200 \mathrm{~K})$ isothermal mesosphere. Such a structure is here again typical of runaway atmospheres, and extensively discussed by, e.g., Goldblatt et al. [2013]: altering $T_{\text {surf }}$ in this regime merely squeezes or expands the thickness of the lowermost unsaturated layer, while most of the thermal emission originating from overlying layers (in the uppermost moist troposphere and lower mesosphere) which are at more or less constant temperatures, hereby keep OLR constant with respect to the surface temperature at Nakajima's limit.

The thermal spectra (with or without taking into account the radiative effect of clouds) originating from the same atmosphere are shown in Figure 8. This spectrum is remarkably independent of surface temperature for $T_{\text {surf }}<T_{\varepsilon}$, and very similar to other runaway atmospheres OLR spectra such as Hamano et al. [2015, their Figure 11] or Goldblatt et al. [2013, their Figure 1]. As could be expected, the spectral features of such atmospheres are dominated by water vapor, with some $\mathrm{CO}_{2}$ absorption bands barely noticeable near 15 and $4.3 \mu \mathrm{m}$. From the spectroscopic analysis of their OLR, these atmospheres cannot be told apart from more evolved 


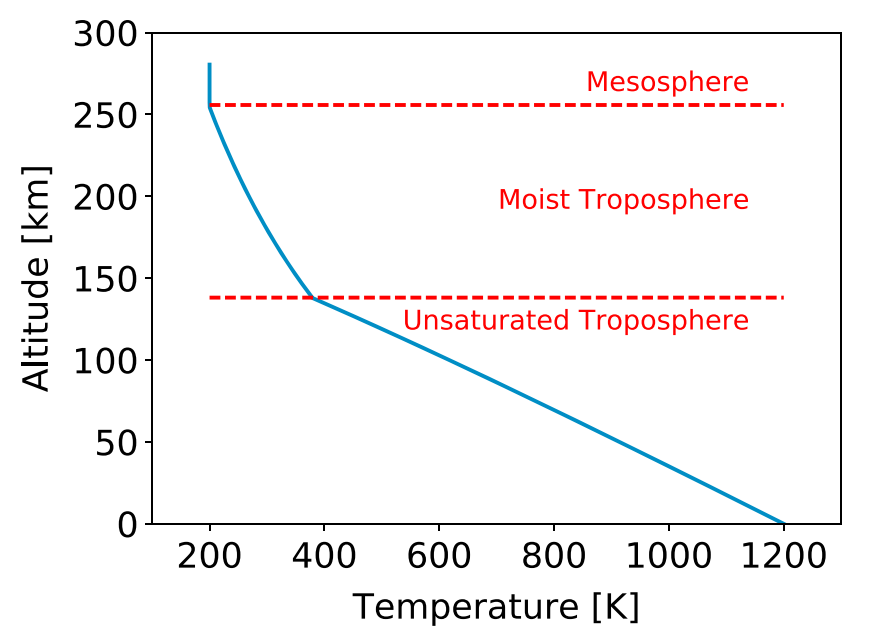

Figure 7. $T(z)$ profile in a $T_{\text {surf }}=1200 \mathrm{~K}<T_{\varepsilon} \approx 1690 \mathrm{~K}$ for $P_{\text {surf }}\left(\mathrm{H}_{2} \mathrm{O}\right)=300$ bar and $P_{\text {surf }}\left(\mathrm{CO}_{2}\right)=100$ bar.

planets at global radiative balance and hosting a thick $\mathrm{H}_{2} \mathrm{O}-\mathrm{CO}_{2}$ atmosphere, somewhat analogous to (a much wetter) Venus. This similarity even extends to featuring near infrared windows (of both $\mathrm{H}_{2} \mathrm{O}$ and $\mathrm{CO}_{2}$ in this case) where the brightness temperature can exceed $400 \mathrm{~K}$ depending on the cloud cover. An extensive discussion on the detectability of such planets can be found in Hamano et al. [2015] for their type I planets, defined as the planets that are able to reach global radiative balance for such relatively low surface temperatures. We further quantitatively discuss the detectability of similar exoplanets in section 3.3.

3.2.2. $T_{\text {surf }}>T_{\varepsilon}$

A typical atmospheric structure in the $T_{\text {surf }}>T_{\varepsilon}$ regime is shown in Figure 9. The vertical extension of the saturated layer is much thinner in this case, and it is also located at much lower pressure levels so that the mass loading (and, therefore, the optical thickness) of the clouds is much lower.

The very high tropopause in these cases results in having most of the thermal emission originating from the hotter, unsaturated troposphere. This enables the OLR to break Nakajima's limit. For example, the spectrum associated with the atmospheric structure shown in Figure 9 is shown in Figure 10. Their brightness temperature for $\lambda>10 \mu \mathrm{m}$ is relatively low (equal to the mesospheric temperature), but most of their thermal emission actually comes from shorter wavelength and concentrated in the few near infrared windows of $\mathrm{H}_{2} \mathrm{O}$ and $\mathrm{CO}_{2}$, below $5 \mu \mathrm{m}$. Also, the optically thin clouds would have little effect on the spectra in such atmospheres. Our cutoff for thermal IR calculations near 1 micron could be challenged here, since the spectrum shown in Figure 10

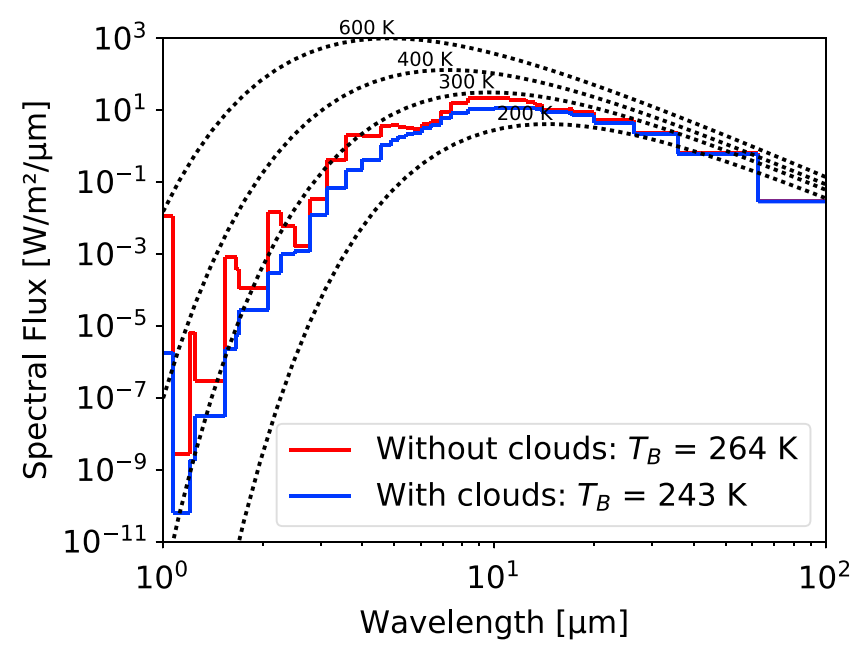

Figure 8. Thermal emission spectrum for the atmosphere shown in Figure 7. The blue spectrum takes into account the radiative effect of clouds, whereas the red spectrum does not. Dotted lines stand for Planck functions at the indicated temperatures. 


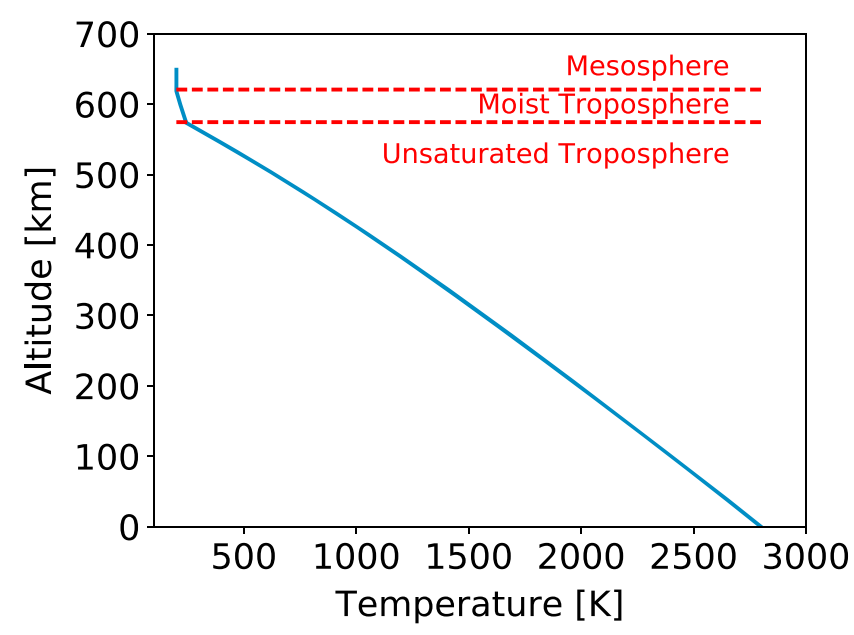

Figure 9. $T(z)$ profile in a $T_{\text {surf }}=2800 \mathrm{~K}>T_{\varepsilon} \approx 1690 \mathrm{~K}$ for $P_{\text {surf }}\left(\mathrm{H}_{2} \mathrm{O}\right)=300$ bar and $P_{\text {surf }}\left(\mathrm{CO}_{2}\right)=100$ bar.

displays significant thermal emission near 1 micron and probably also for shorter wavelength, so that there might be a visible "thermal glow" for such atmospheres, as can be seen in the analogous spectrum of Hamano et al. [2015, their Figure 1a]. However, the resulting underestimation of OLR is found to be small enough, less than a few percents as further detailed in section 4.1.2.

\subsection{Detectability of Magma Ocean Exoplanets}

Considering the spectra shown in Figures 8 and 10, we expect a strong influence of the surface temperature (more specifically the $T_{\text {surf }} / T_{\varepsilon}$ ratio) on the detectability of young exoplanets currently in the magma ocean stage. In order to better quantify this detectability, we define the spectral thermal contrast $C_{i}$ in one of our 36 spectral bands as $C_{i}=\left(R_{p} / R_{*}\right)^{2} \mathrm{OLR}_{i} /\left\langle B\left(T_{*}\right)\right\rangle_{i}$, where $R_{p}$ and $R_{*}$ stand, respectively, for the planet and the host star radii, $\mathrm{OLR}_{i}$ for the planet OLR in a given wave number band, and $\left\langle B\left(T_{*}\right)\right\rangle_{i}$ the Planck function averaged upon the same wave number band for the star temperature $T_{*}$ - that is, assuming that the host star behaves as a blackbody. This contrast underestimates the actual contrast since it does not take into account the reflected stellar component that should be present except for a $180^{\circ}$ phase angle (e.g., during a primary stellar transit).

Results are shown for an Earth-sized planet with a 300 bar $-\mathrm{H}_{2} \mathrm{O}, 100$ bar $-\mathrm{CO}_{2}$ atmosphere around a solar analog $\left(R_{*}=109.2 R_{p}, T_{*}=5772 \mathrm{~K}\right.$, Figure 11) and a red dwarf similar to Proxima Centauri $\left(R_{*}=15.4 R_{p}\right.$, $T_{*}=3042 \mathrm{~K}$, Figure 12). The comparison between both plots shows that the contrast is very much improved for a smaller and cooler star, the larger size of the planet relative to its host star more than offsets the shift of the stellar radiation toward longer wavelengths.

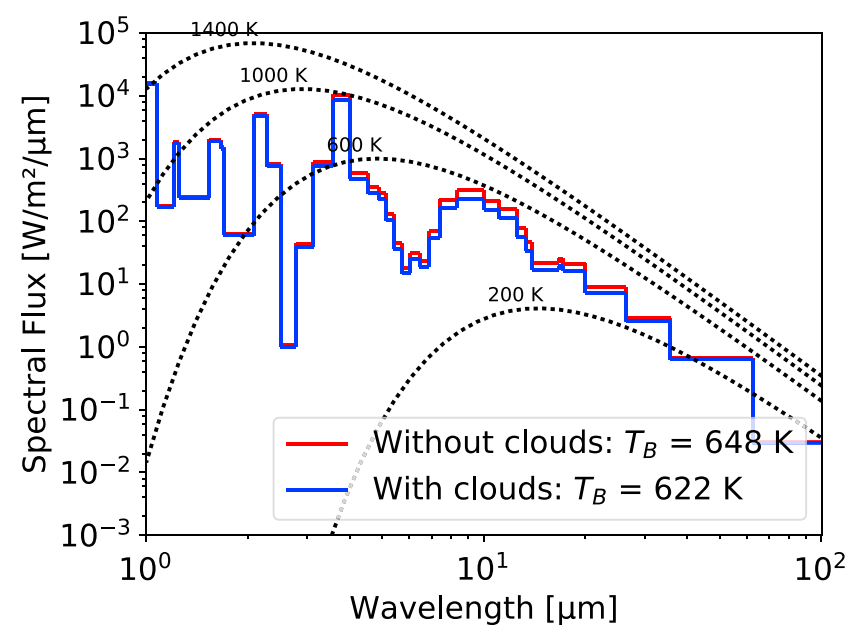

Figure 10. Thermal emission spectrum for the atmosphere shown in Figure 9. Dotted lines stand for Planck functions at the indicated temperatures. 


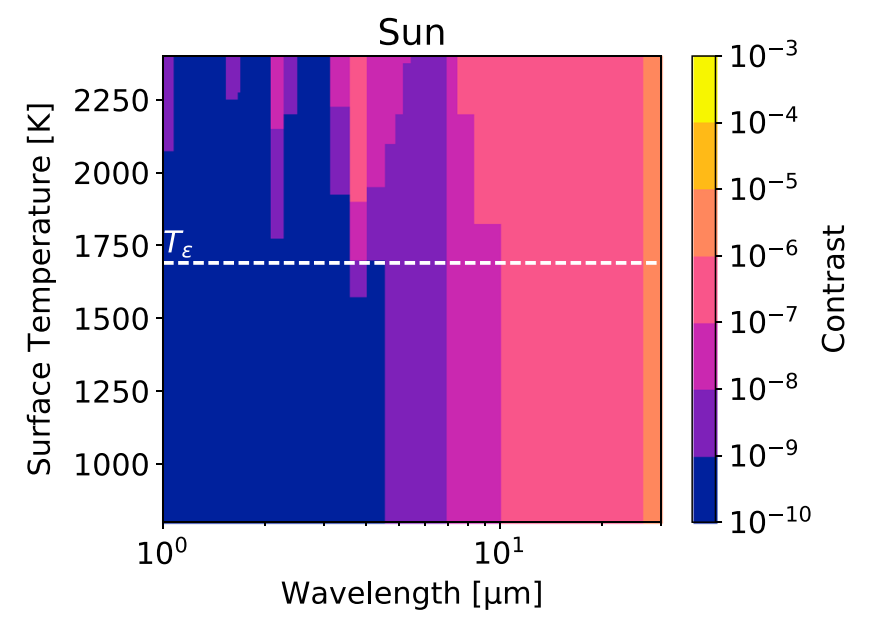

Figure 11. Spectral contrast of the thermal emission of an Earth-sized planet with a 300 bar $\mathrm{H}_{2} \mathrm{O}, 100$ bar- $\mathrm{CO}_{2}$ atmosphere orbiting against a Sun-like star with respect to surface temperature. $T_{\varepsilon} \approx 1690 \mathrm{~K}$ is indicated.

There are two considerations to keep in mind when discussing these contrasts. The first consideration deals, obviously, with the instrumental capabilities available as of 2017 or in the foreseeable future. For example, James Webb Space Telescope (JWST) capabilities as stated by Clampin [2011] gives a minimal contrast in the $10^{-6}-10^{-4}$ range for direct imaging in the near to middle IR. For a single $1 \mathrm{~h}$ transit, the expected JWST photon noise of a $\mathrm{M}$ dwarf near $4 \mu \mathrm{m}$ at a spectral resolution $R \sim 10$ is close to $10^{-5}$ at $10 \mathrm{pc}$ [Turbet et al., 2016, their Figure 14] and decreases with the inverse of the distance. This photon noise would also decrease as the inverse square root of the number of recorded transits, so that for transits also, the $10^{-6}$ to $10^{-4}$ appears a realistic range for our sensitivity. The other, more subtle consideration, deals with the specificity of a detection: are we able to distinguish the thermal emission of a magma ocean planet from the emission of a cooler, older telluric planet?

From the contrasts shown in Figures 11 and 12, it then appears that the highest contrast values (around 10 for the Sun, $>10^{-4}$ for Proxima) are reached for $\lambda>10 \mu \mathrm{m}$. Although well within the range of detectability in the case of a red dwarf star (but only marginally for a Sun-like star), the emission for these longer wavelengths is not very distinctive with respect to surface temperature since emission always originates from the relatively cool mesosphere. Spectral signatures are most distinctive in the spectral windows that "open up" for $T_{\text {surf }}>T_{\varepsilon^{\prime}}$ but these windows lie in the near IR range where the star is much brighter than the planet, thus hampering the resulting contrast, especially for a relatively hot star like the Sun. Therefore, the best diagnostic band is the longest-wavelength spectral window, located in the 3.55-4 $\mu \mathrm{m}$ range (with the $2-2.3 \mu \mathrm{m}$ band as a

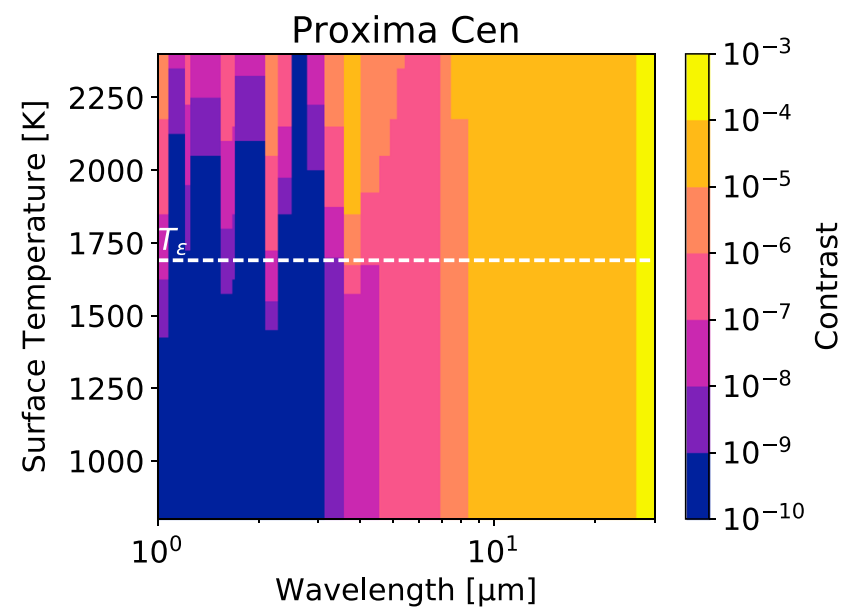

Figure 12. Spectral contrast of the thermal emission of an Earth-sized planet with a 300 bar $\mathrm{H}_{2} \mathrm{O}, 100$ bar $\mathrm{CO}_{2}$ atmosphere orbiting against a Proxima Centauri-like star with respect to surface temperature. $T_{\varepsilon} \approx 1690 \mathrm{~K}$ is indicated. 


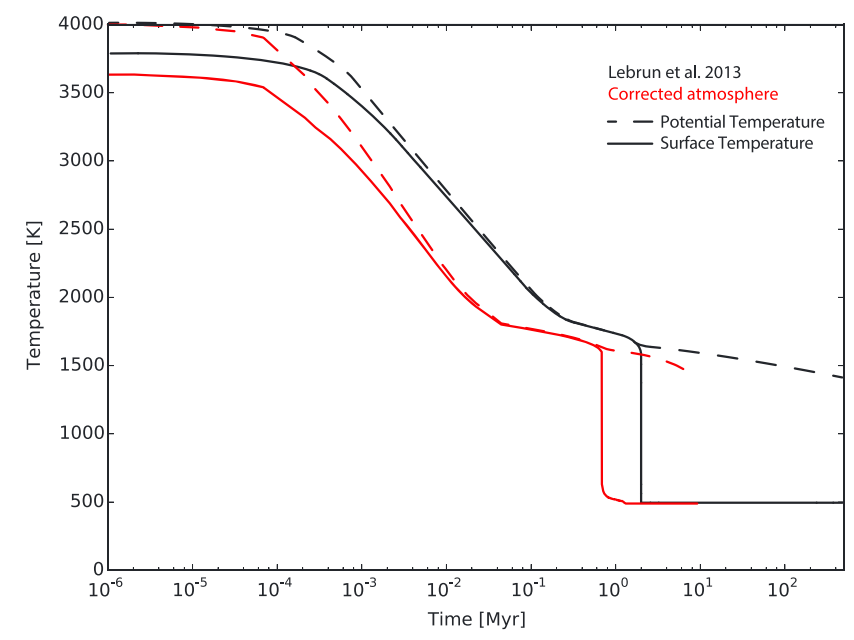

Figure 13. Comparison between the corrected version of the coupled model (in red) and the results of Lebrun et al. [2013] (in black) for a typical run (their Figure 2i, with a planet-Sun distance of $1 \mathrm{AU}, 1.435 \times 10^{-2} \mathrm{wt} \%$ of $\mathrm{CO}_{2}$ and $4.3 \times 10^{-2}$ wt $\%$ of $\mathrm{H}_{2} \mathrm{O}$ ). The solid curves stand for the surface temperatures and the dashed curves for the mantle potential temperatures. The sharp drop in surface temperature indicated that OLR drops below the Nakajima's limit. Therefore, mantle surface can solidify and condensation of an overlying water ocean may occur afterward.

distant second) in a dense $\mathrm{H}_{2} \mathrm{O}-\mathrm{CO}_{2}$ atmosphere. Although detection of the planet emission within this band is unlikely even for a very hot magma ocean planet around a Sun-like star, it would be well within JWST capabilities if a such a magma ocean planet were to orbit a red dwarf within a few hundred parsecs. This optimistic conclusion should however be mitigated by the fact that a Hamano-type I magma ocean planet typically spends less than $10^{5}$ years with $T_{\text {surf }}>T_{\varepsilon}$ (see section 3.4 ), thus drastically limiting the detection time window of such planets. Hamano-type II planets, located close enough to their host stars so that they can reach radiative balance with $T_{\text {surf }}>T_{\varepsilon}$, would remain much longer above the detection threshold, only limited by the gradual escape of their atmospheres, which results in a secular decline of $T_{\varepsilon}$ and therefore of $T_{\text {surf }}$ over millions of years. Finally, it is noteworthy that the only planets that we can hope to detect through their thermal radiation are hot enough $\left(T_{\text {surf }}>T_{\varepsilon}\right)$ to radiate in their NIR windows regardless of their expected cloud cover according to our cloud model, since these clouds lie at such high altitudes to be optically thin in the NIR range.

\subsection{Coupled Evolution of the Atmosphere and Magma Ocean}

The atmospheric code developed by Marcq [2012] was one of the building blocks of the model developed by Lebrun et al. [2013] to study the coupled evolution of the atmosphere and magma ocean (MO) of an Earth-sized planet. We therefore checked how its results could be influenced by this update to the atmospheric model and correction of the OLR calculation. Figure 13 presents a comparison of the potential and surface temperatures calculated by the two versions of the code, for the conditions shown in Lebrun et al. [2013, their Figure 3i]. Only the cooling time scale differs between the two versions: in the new updated version, the MO cools down faster but reaches the same temperature at the end of the liquid MO phase. The MO solidification is accelerated by a factor of about $2.5\left(8 \cdot 10^{5}\right.$ versus $2 \cdot 10^{6}$ years, respectively), which is consistent with the fact that the corrected NL is now about twice the value from Marcq [2012]. However, this significant correction does not challenge the results about the surface conditions at the end of the MO phase obtained by Lebrun et al. [2013]. Their conclusions about the existence of two types of planets, one condensing water at the end of the MO phase and the other remaining too hot to do so (so-called Hamano-type I and type II planets) are therefore still valid. More results will be presented by Salvador et al. [2017], notably extending the range of investigated volatile inventories.

\section{Discussion}

\subsection{Limitations}

Our model is already functional and can be applied to a wide range of planetary atmospheres. There are however some caveats that should be kept in mind before applying this model beyond its validity domain.

\subsubsection{Spherical Corrections}

When modeling very hot atmospheres $\left(T_{\text {surf }}>2500 \mathrm{~K}\right)$ around smaller (Mars-sized) telluric planets, we find that the atmospheric scale height $H$ is not negligible compared to the planetary radius $R$. This challenges the 
common plane-parallel assumption of most models, including ours. However, some corrections can solve or at least alleviate these potential sphericity issues.

First, hydrostatic equilibrium itself is unaffected by sphericity (1-D spherical gradient is $\partial P / \partial r$ instead of $\partial P / \partial z$ in Cartesian coordinates), but the straightfoward relation between surface pressure and atmospheric mass needed by interior models [Lebrun et al., 2013; Salvador et al., 2017] is lost. However, numerical radial integration of the density profile is straightforward and yields the required volatile mass.

Adaptations to the radiative transfer code are more complex. We found no publicly available 1-D spherical radiative transfer codes similar to DISORT; Rannou et al. [2010] however provided us with a pseudospherical adaptation of DISORT that takes spherical geometry into consideration only for the incident and emerging beams. This could be used in a future version of our model, provided the mean free path of photons is small compared to the planetary radius. In such a case, a first-order approximation could be to correct the OLR - in $\mathrm{W} / \mathrm{m}^{2}$, the area being understood at the planetary surface - for the actual altitude where the thermal radiation originates. One way to do so, especially in the case of negligible scattering, would be, for each $g$ bin in the $k$-correlated code, to locate the altitude $z_{g}$ where the physical temperature is equal to the brightness temperature (as computed with the plane-parallel radiative code), and weighting the corresponding spectral flux by $\left(1+z_{g}{ }^{2} / R^{2}\right)$ before integration on the $g$ space. This would enhance the OLR, as well as lead to less intuitive consequences if the effective altitude of thermal emission differs significantly from, e.g., the effective altitude for the absorption of the stellar component (see section 4.1.2). Similar arguments are developed by Goldblatt [2015], and result in an OLR enhancement on the order of $10 \mathrm{~W} / \mathrm{m}^{2}$ for pure steam atmospheres around Earth-sized planets (up to $30 \mathrm{~W} / \mathrm{m}^{2}$ for Mars-sized planets).

A last spherical correction would deal with the mesospheric temperature: if we eventually compute it through canceling the divergence of the OLR + absorbed stellar flux at vanishing optical depth, we must keep in mind that the radial divergence operator in spherical coordinates is not identical to the vertical divergence in Cartesian coordinates (unlike the vertical and radial gradients discussed earlier in this section). The net effect would be a cooling of the mesosphere - the outer surface of the topmost spherical shell cooling to space being larger than the inner surface heated from below in spherical geometry, but the exact extent of this extra cooling is yet to be quantified.

\subsubsection{Radiative Shortcomings}

As already mentioned before (section 3.2.2), we need in the near future to extend the radiative transfer calculations to shorter wavelengths whenever considering very hot surfaces surrounded with comparatively light atmospheres - this was already highlighted and extensively discussed by Marca [2012]. Such an extension would encompass visible and near UV (up to $35,000 \mathrm{~cm}^{-1}$ ) so that we could model absorption and scattering of the stellar component for stars in the $\mathrm{G}, \mathrm{K}$, and $\mathrm{M}$ spectral classes.

Incidentally, the inclusion of shorter wavelengths in our thermal radiative transfer calculations would solve a possible issue in the case of very hot surfaces under very thin atmospheres, where thermal radiation shortward of $1 \mu \mathrm{m}$ is expected to be significant. However, this is not the case in the parameter space covered in this paper: graphical integration performed upon Hamano et al. [2015, their Figure 1a] spectrum shows that for a relatively hot $2500 \mathrm{~K}$ surface under a relatively thin 50 bar pure steam atmosphere, OLR shortward of $1 \mu \mathrm{m}$ only accounts for less than $5 \%$ of the total OLR.

This inclusion would yield considerable improvements in our model, namely, (1) a true computation of the spectral and bolometric albedo of these atmospheres using the existing radiative transfer code and (2) as a consequence, open the possibility to improve the computation of the $T(p)$ profile in the upper radiative layers of the atmosphere better than the current isothermal assumption. Of course, Rayleigh scattering would have to be added to the Mie scattering which is the only one considered in the current stage of the model.

Spherical corrections to the stellar component of the radiative transfer would be similar to what is proposed in section 4.1.1: weighting in each $g$ bin according to the altitude of effective scattering, defined by a nadir optical depth $\bar{\tau}_{\text {scat }}=\varpi_{0}\left[3\left(1-\varpi_{0}\right)\right]^{-1 / 2}$ in an analytical semi-infinite two-stream solution, where $\varpi_{0}$ is the single scattering albedo.

\section{Conclusion}

Our updated model mostly confirms the qualitative findings from Marcq [2012] and comparable studies such as Hamano et al. [2013]. Including this updated model into the coupled model of Lebrun et al. [2013] also 
Table 1. Comparison Between Recent $\mathrm{H}_{2} \mathrm{O}$-Rich Atmospheric Models

\begin{tabular}{lccccc} 
Reference & Composition & Rad. Transfer & $\mathrm{NL}\left(\mathrm{W} / \mathrm{m}^{2}\right)$ & Clouds & Coupling \\
\hline Goldblatt et al. [2013] & $\mathrm{H}_{2} \mathrm{O}$-air & line-by-line & 282 & None & None \\
Leconte et al. [2013] & $\mathrm{H}_{2} \mathrm{O}$-air & $k$-correlated & 282 & Yes & 3-D general circulation model \\
Kopparapu et al. [2013] & $\mathrm{H}_{2} \mathrm{O}-\mathrm{CO}_{2}$ & $k$-correlated & 291 & None & None \\
Kopparapu et al. [2014] & $\mathrm{H}_{2} \mathrm{O}-\mathrm{N}_{2}$ & $k$-correlated & $280^{\mathrm{a}}$ & None & None \\
Hamano et al. [2013] & $\mathrm{H}_{2} \mathrm{O}$ & Gray & 300 & None & Interior \& Escape \\
Hamano et al. [2015] & $\mathrm{H}_{2} \mathrm{O}$ & line-by-line & 280 & None & Interior \& Escape \\
Marcq [2012] & $\mathrm{H}_{2} \mathrm{O}-\mathrm{CO}_{2}$ & $>1 \mu \mathrm{m}, k$-corr. & 200 & Optional & Interior [Lebrun et al., 2013] \\
This study & $\mathrm{H}_{2} \mathrm{O}-\mathrm{CO}_{2}\left(-\mathrm{N}_{2}\right)$ & $>1 \mu \mathrm{m}, k$-corr. & 280 & Optional & Interior [Salvador et al., 2017] \\
\hline
\end{tabular}

${ }^{\mathrm{a}}$ For an Earth-mass planet.

confirms its qualitative behavior, as can be seen in Figure 13. The main correction deals with the new estimation of Nakajima's limit in the clear sky case around $280 \mathrm{~W} / \mathrm{m}^{2}$, much better in line with comparable recent work (Table 1). The improved numerical stability and switch to an adaptative $p$ grid instead of a fixed $z$ grid also enabled the investigation of a broader input parameter space, so that we could derive an analytic parameterization of the threshold blanketing temperature (defined as the surface temperature where the effective emissivity peaks) with respect to the surface pressures of $\mathrm{H}_{2} \mathrm{O}$ and $\mathrm{CO}_{2}$. The new updated coupled model is used by Salvador et al. [2017] to systematically investigate the influence of volatile content on the inner limit of water condensation at the end of the magma ocean phase.

We can also model the effect of a $100 \%$, thick cloud cover, leading to a reduction of the OLR by up to $40 \%$ and a raise of about $10 \%$ of the threshold blanketing temperature. However, since a proper model of the cloud cover requires a more sophisticated 3-D model like in Leconte et al. [2013], we chose to focus most of our studies on the cloudless case. Current limitations (near IR cutoff, plane-parallel assumption, and lack of stellar radiative transfer) and some hints about how to overcome them were also discussed.

These updates to the model of Marcq [2012] are best understood in the context of recent comparable work for $\mathrm{H}_{2} \mathrm{O}$-rich atmospheres, whether around magma ocean planets or for runaway greenhouse studies (see Table 1). Their similarities, far from being redundant, enable detailed cross comparison and provide useful benchmarking during the development or adaptation of new models. We can also see that no model is superior in all points to the others: they all have different strengths and weaknesses, which enables any final user developing their own coupled atmosphere-interior-escape model to select the one best suited to their needs.

Acknowledgments

We wish to thank M. Turbet, J. Leconte, C. Goldblatt, and F. Selsis for fruitful scientific discussions while presenting this model. The authors have been supported by a grant from the Programme National de Planétologie (PNP) sponsored by the Institut National des Sciences de I'Univers (INSU). Arnaud Salvador's PhD is supported by a grant from the Ministère de la Recherche and Université Paris Sud. Data policy: the source code of this model can be downloaded here: http://marcq. page.latmos.ipsl.fr/radconv1d.html.

\section{References}

Abe, Y., and T. Matsui (1988), Evolution of an impact-generated $\mathrm{H}_{2} \mathrm{O}-\mathrm{CO}_{2}$ atmosphere and formation of a hot proto-ocean on Earth, J. Atmos. Sci., 45, 3081-3101, doi:10.1175/1520-0469(1988)045<3081:EOAIGH>2.0.CO;2.

Bézard, B., A. Fedorova, J.-L. Bertaux, A. Rodin, and O. Korablev (2011), The 1.10- and 1.18- $\mu \mathrm{m}$ nightside windows of Venus observed by SPICAV-IR aboard Venus Express, Icarus, 216, 173-183, doi:10.1016/j.icarus.2011.08.025.

Chase, M. (1998), Nist-JANAF Thermochemical Tables, Am. Chem. Soc., Washington, D. C.

Clampin, M. (2011), The James Webb space telescope and its capabilities for exoplanet science, in The Astrophysics of Planetary Systems: Formation, Structure, and Dynamical Evolution, IAU Symposium, vol. 276, edited by A. Sozzetti, M. G. Lattanzi, and A. P. Boss, pp. 335-342, Cambridge Univ. Press, Cambridge, U. K., doi:10.1017/S1743921311020400.

Clough, S. A., M. W. Shephard, E. J. Mlawer, J. S. Delamere, M. J. lacono, K. Cady-Pereira, S. Boukabara, and P. D. Brown (2005), Atmospheric radiative transfer modeling: A summary of the AER codes, J. Quant. Spectrosc. Radiat. Transfer, 91, 233-244, doi:10.1016/j.jqsrt.2004.05.058.

Elkins-Tanton, L. T. (2008), Linked magma ocean solidification and atmospheric growth for Earth and Mars, Earth Planet. Sci. Lett., 271, $181-191$, doi:10.1016/j.epsl.2008.03.062.

Eymet, V., C. Coustet, and B. Piaud (2016), KSPECTRUM: An open-source code for high-resolution molecular absorption spectra production, J. Phys. Conf. Ser., 676(1), 12005, doi:10.1088/1742-6596/676/1/012005.

Goldblatt, C. (2015), Habitability of waterworlds: Runaway greenhouses, atmospheric expansion, and multiple climate states of pure water atmospheres, Astrobiology, 15, 362-370, doi:10.1089/ast.2014.1268.

Goldblatt, C., T. D. Robinson, K. J. Zahnle, and D. Crisp (2013), Low simulated radiation limit for runaway greenhouse climates, Nat. Geosci., 6 , 661-667, doi:10.1038/ngeo1892.

Haar, L., J. Gallagher, G. Kell, and National Standard Reference Data System (U.S.) (1984), NBS/NRC Steam Tables: Thermodynamic and Transport Properties and Computer Programs for Vapor and Liquid States of Water in SI Units, Hemisphere Publ. Corp., Washington, D. C.

Hamano, K., Y. Abe, and H. Genda (2013), Emergence of two types of terrestrial planet on solidification of magma ocean, Nature, 497, 607-610, doi:10.1038/nature12163.

Hamano, K., H. Kawahara, Y. Abe, M. Onishi, and G. L. Hashimoto (2015), Lifetime and spectral evolution of a magma ocean with a steam atmosphere: Its detectability by future direct imaging, Astrophys. J., 806, 216, doi:10.1088/0004-637X/806/2/216. 
Ingersoll, A. P. (1969), The runaway greenhouse: A history of water on Venus, J. Atmos. Sci., 26, 1191-1198, doi:10.1175/1520-0469(1969)026<1191:TRGAHO>2.0.CO;2.

Kasting, J. F. (1988), Runaway and moist greenhouse atmospheres and the evolution of Earth and Venus, Icarus, 74, 472-494, doi:10.1016/0019-1035(88)90116-9.

Komabayashi, M. (1967), Discrete equilibrium temperatures of a hypothetical planet with the atmosphere and the hydrosphere of one component-two phase system under constant solar radiation, J. Meteorol. Soc. Jpn., 45, 137-139.

Kopparapu, R. K., R. Ramirez, J. F. Kasting, V. Eymet, T. D. Robinson, S. Mahadevan, R. C. Terrien, S. Domagal-Goldman, V. Meadows, and R. Deshpande (2013), Habitable zones around main-sequence stars: New estimates, Astrophys. J., 765, 131, doi:10.1088/0004-637X/765/2/131.

Kopparapu, R. K., R. M. Ramirez, J. SchottelKotte, J. F. Kasting, S. Domagal-Goldman, and V. Eymet (2014), Habitable zones around main-sequence Stars: Dependence on planetary mass, Astrophys. J. Lett., 787, L29, doi:10.1088/2041-8205/787/2/L29.

Kopparapu, R. k., E. T. Wolf, J. Haqq-Misra, J. Yang, J. F. Kasting, V. Meadows, R. Terrien, and S. Mahadevan (2016), The inner edge of the habitable zone for synchronously rotating planets around low-mass stars using general circulation models, Astrophys. J., 819, 84, doi:10.3847/0004-637X/819/1/84

Lebrun, T., H. Massol, E. Chassefière, A. Davaille, E. Marcq, P. Sarda, F. Leblanc, and G. Brandeis (2013), Thermal evolution of an early magma ocean in interaction with the atmosphere, J. Geophys. Res. Planets, 118, 1155-1176, doi:10.1002/jgre.20068.

Leconte, J., F. Forget, B. Charnay, R. Wordsworth, and A. Pottier (2013), Increased insolation threshold for runaway greenhouse processes on Earth-like planets, Nature, 504, 268-271, doi:10.1038/nature12827.

Lupu, R. E., K. Zahnle, M. S. Marley, L. Schaefer, B. Fegley, C. Morley, K. Cahoy, R. Freedman, and J. J. Fortney (2014), The atmospheres of Earthlike planets after giant impact events, Astrophys. J., 784, 27, doi:10.1088/0004-637X/784/1/27.

Ma, Q., and R. H. Tipping (1992), A far wing line shape theory and its application to the foreign-broadened water continuum absorption. III, J. Chem. Phys., 97, 818-828, doi:10.1063/1.463184.

Marcq, E. (2012), A simple 1-D radiative-convective atmospheric model designed for integration into coupled models of magma ocean planets, J. Geophys. Res., 117, E01001, doi:10.1029/2011JE003912.

Marcq, E., B. Bézard, P. Drossart, G. Piccioni, J. M. Reess, and F. Henry (2008), A latitudinal survey of CO, OCS, $\mathrm{H}_{2} \mathrm{O}$, and $\mathrm{SO}_{2}$ in the lower atmosphere of Venus: Spectroscopic studies using VIRTIS-H, J. Geophys. Res., 113, E00B07, doi:10.1029/2008JE003074.

Nakajima, S., Y.-Y. Hayashi, and Y. Abe (1992), A study on the 'runaway greenhouse effect' with a one-dimensional radiative-convective equilibrium model, J. Atmos. Sci., 49, 2256-2266, doi:10.1175/1520-0469(1992)049<2256:ASOTGE>2.0.CO;2.

Rannou, P., T. Cours, S. Le Mouélic, S. Rodriguez, C. Sotin, P. Drossart, and R. Brown (2010), Titan haze distribution and optical properties retrieved from recent observations, Icarus, 208, 850-867, doi:10.1016/j.icarus.2010.03.016.

Salvador, A., H. Massol, A. Davaille, E. Marcq, P. Sarda, and E. Chassefière (2017), The relative influence of $\mathrm{H}_{2} \mathrm{O}$ and $\mathrm{CO}_{2}$ on the primitive surface conditions and evolution of rocky planets, J. Geophys. Res. Planets, 122, doi:10.1002/2017JE005286.

Schaefer, L., R. D. Wordsworth, Z. Berta-Thompson, and D. Sasselov (2016), Predictions of the atmospheric composition of GJ 1132b, Astrophys. J., 829, 63, doi:10.3847/0004-637X/829/2/63.

Stamnes, K., S.-C. Tsay, K. Jayaweera, and W. Wiscombe (1988), Numerically stable algorithm for discrete-ordinate-method radiative transfer in multiple scattering and emitting layered media, Appl. Opt., 27, 2502-2509, doi:10.1364/AO.27.002502.

Turbet, M., J. Leconte, F. Selsis, E. Bolmont, F. Forget, I. Ribas, S. N. Raymond, and G. Anglada-Escudé (2016), The habitability of Proxima Centauri b. II. Possible climates and observability, Astrono. Astrophys., 596, A112, doi:10.1051/0004-6361/201629577.

Wordsworth, R. D., and R. T. Pierrehumbert (2013), Water loss from terrestrial planets with $\mathrm{CO}_{2}$-rich atmospheres, Astrophys. J., 778, 154, doi:10.1088/0004-637X/778/2/154.

Yang, J., and D. S. Abbot (2014), A low-order model of water vapor, clouds, and thermal emission for tidally locked terrestrial planets, Astrophys. J., 784, 155, doi:10.1088/0004-637X/784/2/155.

Yang, J., N. B. Cowan, and D. S. Abbot (2013), Stabilizing cloud feedback dramatically expands the habitable zone of tidally locked planets, Astrophys. J. Lett., 771, L45, doi:10.1088/2041-8205/771/2/L45.

Yang, J., G. Boué, D. C. Fabrycky, and D. S. Abbot (2014), Strong dependence of the inner edge of the habitable zone on planetary rotation rate, Astrophys. J. Lett., 787, L2, doi:10.1088/2041-8205/787/1/L2. 